Pacific Journal of 


\title{
DERIVATIONS ON THE LINE AND FLOWS ALONG ORBITS
}

\author{
C. J. K. BATTY
}

The closure of the derivation $\lambda D: C_{c}^{1}(\mathbb{R}) \rightarrow C_{0}(\mathbb{R})$ defined by $(\lambda D)(f)=\lambda f^{\prime}$, where $\lambda: \mathbb{R} \rightarrow \mathbb{R}$ is continuous, generates a $C_{0}$-group on $C_{0}(\mathbb{R})$ (corresponding to a flow on $\mathbb{R}$ ) if and only if $1 / \lambda$ is not locally integrable on either side of any zero of $\lambda$ or at $\pm \infty$.

If $S$ is a flow on a locally compact, Hausdorf, space $X$ with fixed point set $X_{S}^{0}, \delta_{S}$ is the generator of the induced action on $C_{0}(X), \lambda$ : $X \backslash X_{S}^{0} \rightarrow \mathbb{R}$ is continuous, and bounded on sets of low frequency under $S$, and $t \rightarrow \lambda\left(S_{t} \omega\right)^{-1}$ is not locally integrable on either side of any zero or at $\pm \infty$, then the flows along the orbits of $S$ form a flow on $X$ whose generator acts as $\lambda \delta_{S}$.

1. Introduction. Let $S$ be a flow on a locally compact, Hausdorff, space $X$, and $\delta_{S}$ be the generator of the associated one-parameter group of *-automorphisms of $C_{0}(X)$, the commutative $C^{*}$-algebra of continuous complex-valued functions on $X$ which vanish at infinity. Thus

$$
\delta_{S} f=\lim _{t \rightarrow 0} t^{-1}\left(f \circ S_{t}-f\right)
$$

whenever the limit exists (pointwise, and hence uniformly) and defines a function in $C_{0}(X)$. Let $\mathscr{D}_{S}^{\infty}=\bigcap_{n \geq 1} \mathscr{D}\left(\delta_{S}^{n}\right)$. Then $\mathscr{D}_{S}^{\infty}$ is a dense *-subalgebra of $C_{0}(X)$. If $\delta: \mathscr{D}_{S}^{\infty} \rightarrow C_{0}(X)$ is a *-derivation, then there is a function $\lambda: X \rightarrow \mathbb{R}$ such that

$$
\delta f=\lambda \delta_{S} f \quad\left(f \in \mathscr{D}_{S}^{\infty}\right)
$$

[1]. The function $\lambda$ may be chosen arbitrarily on the fixed point set $X_{S}^{0}$ :

$$
\begin{aligned}
X_{S}^{0} & =\left\{\omega \in X: S_{t} \omega=\omega \text { for all } t\right\} \\
& =\left\{\omega \in X: \delta_{S} f(\omega)=0 \text { for all } f \text { in } \mathscr{D}_{S}^{\infty}\right\},
\end{aligned}
$$

and we shall always assume that $\lambda=0$ on $X_{S}^{0}$. However, $\lambda$ is uniquely determined and continuous on $X \backslash X_{S}^{0}$, and satisfies a bound of the form

$$
|\lambda(\omega)| \leq c\left(1+\nu(\omega)^{n}\right) \quad\left(\omega \in X \backslash X_{S}^{0}\right)
$$

for some constant $c \geq 0$, and integer $n \geq 0$, where $\nu(\omega)$ is the frequency of $\omega$, so

$$
\nu(\omega)^{-1}=\inf \left\{t>0: S_{t} \omega=\omega\right\}
$$

$(\nu(\omega)=0$ if $\omega$ is aperiodic) (see [4]). 
We shall therefore study the *-derivations $\lambda \delta_{S}$ defined by

$$
\left(\lambda \delta_{S}\right) f= \begin{cases}\lambda \delta_{S} f & \text { on } X \backslash X_{S}^{0} \\ 0 & \text { on } X_{S}^{0}\end{cases}
$$

whenever the right-hand side defines a function in $C_{0}(X)$. Here $\lambda$ : $X \backslash X_{S}^{0} \rightarrow \mathbb{R}$ is a continuous function. The domain $\mathscr{D}\left(\lambda \delta_{S}\right)$ contains $\mathscr{D}_{S}^{\infty}$ if and only if $\lambda$ satisfies a bound of the form $(*)$, but this will not necessarily be assumed. Nevertheless, $\mathscr{D}\left(\lambda \delta_{S}\right)$ is always reasonably large. Indeed for any $\omega$ in $X \backslash X_{S}^{0}, \varepsilon>0$ such that $2 \varepsilon \nu(\omega)<1$ and $F$ in $C^{\infty}[-\varepsilon, \varepsilon]$, there exists $f$ in $\mathscr{D}_{S}^{\infty}$ such that $f\left(S_{t} \omega\right)=F(t)(|t| \leq \varepsilon)$, and $\operatorname{supp} f \subset X \backslash X_{S}^{0}$ [4]. In particular, $f \in \mathscr{D}\left(\lambda \delta_{S}\right)$.

The properties of interest are whether there is a flow $T$ whose generator $\delta_{T}$ extends $\lambda \delta_{S}$, and if so whether $T$ is unique and whether $\mathscr{D}\left(\lambda \delta_{S}\right)$ (or some smaller subalgebra) is a core for $\delta_{T}$. Considering both functions which vary transversally and along the orbits of $S$, it is apparent that $T$ should be a flow along the orbits of $S$ whose speed is given at each point by the function $\lambda$. Thus

$$
T_{t} S_{s} \omega=S_{\tau_{\omega}(s, t)} \omega
$$

where $\tau_{\omega}$ is a flow on $\mathbb{R}$ such that

$$
\partial \tau_{\omega} / \partial t=\lambda_{\omega} \circ \tau_{\omega}
$$

where $\lambda_{\omega}(s)=\lambda\left(S_{s} \omega\right)$.

The first stage (\$2) therefore is to study flows $T$ on $\mathbb{R}$ satisfying the differential equation

$$
\partial T / \partial t=\lambda \circ T
$$

where $\lambda: \mathbb{R} \rightarrow \mathbb{R}$ is a continuous function. If $1 / \lambda$ is not locally integrable on either side of any zero of $\lambda$ or at $\pm \infty$, then there is a unique flow $T$ of this type, each zero of $\lambda$ is a fixed point of $T$, and $C_{c}^{\infty}(\mathbb{R})$ is a core for $\delta_{T}$. Otherwise, there may be no flows or there may be many flows.

In $\S 3$, it is shown that if each $\lambda_{\omega}$ satisfies these conditions of reciprocal non-integrability, then the flows with speeds $\lambda_{\omega}$ along the orbits together define a flow on $X$ whose generator extends $\lambda \delta_{S}$.

There is some overlap between $\S 2$ of this paper, a paper of de Laubenfels [6], which left several questions incompletely answered, and an unpublished manuscript of the author's [2] which has circulated and been cited quite widely. The results of $\S 3$ are more general than those obtained in $[3,7]$, where it was assumed that $\lambda$ satisfies a Lipschitz condition

$$
\left|\lambda\left(S_{t} \omega\right)-\lambda(\omega)\right| \leq|t| \kappa(\nu)
$$


whenever $\nu(\omega) \leq \nu$. Such a condition implies the reciprocal non-integrability conditions.

I am grateful to $\mathrm{R}$. de Laubenfels for his helpful response to my queries concerning [6], and to D. W. Robinson for his encouragement in reviving this subject while I was visiting the Australian National University at his invitation.

2. The real line. Sakai [9] has raised the question of characterizing all flows $T$ on $[0,1]$ whose generator extends $\lambda D$, where $\lambda \in C[0,1]$ and $D$ denotes differentiation defined on $C^{1}[0,1]$. The motivation for this was the fact that, for any flow $T$ on $[0,1]$, there is a homeomorphism $\theta$ of $[0,1]$ such that $\delta_{\theta T \theta^{-1}}$ extends $\lambda D$ for some $\lambda$. Similar remarks apply to flows on $\mathbb{R}$, where $D$ itself is the generator for the flow of translations, and we shall work on the whole line, at least initially.

In fact, one can, by choosing $\theta$ appropriately, arrange that $\theta T \theta^{-1}$ is one of the flows $T_{U}^{\varepsilon}$ described in the following example [10, p. 26]. But this fact does not directly help to decide when $\lambda D$ extends to a generator, nor is it helpful in considering flows on general spaces.

EXAMPLE 2.1. For each open interval $I$ in $\mathbb{R}$, define flows $T_{I}$ on $I$ as follows:

$$
\begin{aligned}
T_{(a, b)}(x, t) & =\frac{b(x-a) e^{(b-a) t}+a(b-x)}{b-x+(x-a) e^{(b-a) t}}, \\
T_{(a, \infty)}(x, t) & =a+(x-a) e^{t}, \\
T_{(-\infty, b)}(x, t) & =b+(x-b) e^{-t}, \\
T_{\mathbb{R}}(x, t) & =x+t .
\end{aligned}
$$

Now let $U$ be an open subset of $\mathbb{R}, \mathscr{C}_{U}$ be the set of all connected components of $U$, and $\varepsilon$ be a function of $\mathscr{C}_{U}$ into $\{-1,1\}$. Define

$$
T_{U}^{\varepsilon}(x, t)= \begin{cases}T_{I}(x, \varepsilon(I) t) & \left(x \in I \in \mathscr{C}_{U}\right), \\ x & (x \in \mathbb{R} \backslash U) .\end{cases}
$$

Then $T_{U}^{\varepsilon}$ is a flow on $\mathbb{R}$, and its generator is the closure of $\lambda_{U}^{\varepsilon} D \mid C_{c}^{\infty}(\mathbb{R})$, where

$$
\lambda_{U}^{\varepsilon}(x)= \begin{cases}\varepsilon((a, b))(x-a)(b-x) & \left(x \in(a, b) \in \mathscr{C}_{U}\right), \\ \varepsilon((a, \infty))(x-a) & \left(x \in(a, \infty) \in \mathscr{C}_{U}\right), \\ \varepsilon((-\infty, b))(b-x) & \left(x \in(-\infty, b) \in \mathscr{C}_{U}\right), \\ \varepsilon(\mathbb{R}) & (\text { if } U=\mathbb{R}), \\ 0 & (x \in \mathbb{R} \backslash U) .\end{cases}
$$


Let $\lambda: \mathbb{R} \rightarrow \mathbb{R}$ be any continuous function, and put

$$
\begin{aligned}
& Z(\lambda)=\{x \in \mathbb{R}: \lambda(x)=0\}, \\
& U(\lambda)=\mathbb{R} \backslash Z(\lambda)=\{x: \lambda(x) \neq 0\} .
\end{aligned}
$$

For $x$ in $U(\lambda)$, let

$$
\begin{aligned}
& \alpha_{x}=\sup \{y<x: \lambda(y)=0\}, \\
& \beta_{x}=\inf \{y>x: \lambda(y)=0\}
\end{aligned}
$$

with the convention that the supremum of the empty set is $-\infty$, and the infimum is $+\infty$.

Let $A_{l}^{+}(\lambda)$ (respectively, $A_{l}^{-}(\lambda)$ ) be the set of all points $x$ in $Z(\lambda) \cup$ $\{\infty\}$ such that for some $y<x, \lambda \geq 0$ (respectively, $\lambda \leq 0)$ in $(y, x)$ and $1 / \lambda$ is integrable over $(y, x)$. Let $A_{r}^{+}(\lambda)$ (respectively, $A_{r}^{-}(\lambda)$ ) be the set of all $x$ in $Z(\lambda) \cup\{-\infty\}$ such that for some $z>x, \lambda \geq 0$ (respectively, $\lambda \leq 0)$ in $(x, z)$ and $1 / \lambda$ is integrable over $(x, z)$. Let

$$
\begin{gathered}
A_{l}(\lambda)=A_{l}^{+}(\lambda) \cup A_{l}^{-}(\lambda), \quad A_{r}(\lambda)=A_{r}^{+}(\lambda) \cup A_{r}^{-}(\lambda), \\
A(\lambda)=A_{l}(\lambda) \cup A_{r}(\lambda) .
\end{gathered}
$$

The first lemma specifies the properties which amount to a flow on $\mathbb{R}$ having speed $\lambda$. The proof is elementary and will be omitted.

LEMMA 2.2. Let $T$ be a flow on $\mathbb{R}$, and $\lambda: \mathbb{R} \rightarrow \mathbb{R}$ be continuous. The following are equivalent:

(i) $T$ is differentiable with respect to $t$, and $\partial T / \partial t=\lambda \circ T$,

(ii) $C_{c}^{\infty}(\mathbb{R}) \subset \mathscr{D}\left(\delta_{T}\right)$ and $\delta_{T}$ extends $\lambda D \mid C_{c}^{\infty}(\mathbb{R})$,

(iii) $C_{c}^{1}(\mathbb{R}) \subset \mathscr{D}\left(\delta_{T}\right)$ and $\delta_{T}$ extends $\lambda D \mid C_{c}^{1}(\mathbb{R})$,

(iv) If $x \in U(\lambda)$ and $T_{t} x \in\left(\alpha_{x}, \beta_{x}\right)$, then

$$
\int_{x}^{T_{t} x} \frac{d y}{\lambda(y)}=t
$$

if $x \in \operatorname{int} Z(\lambda)$, then $T_{t} x=x$.

COROLlary 2.3. Let $T$ be a flow with speed $\lambda$ (so that $T$ satisfies the conditions of Lemma 2.2) and $x \in U_{\lambda}$. The following are equivalent:

(i) $\left\{T_{t} x: t \in \mathbb{R}\right\} \subset U(\lambda)$,

(ii) $\left\{T_{t} x: t \in \mathbb{R}\right\}=\left(\alpha_{x}, \beta_{x}\right)$,

(iii) $\alpha_{x} \notin A_{r}(\lambda)$ and $\beta_{x} \notin A_{l}(\lambda)$.

The following result (for $[0,1]$ rather than $\mathbb{R}$ ) was included in [6], but no proof was given of the core property. The construction of $T$ appeared earlier in [11]. 
THEOREM 2.4. Let $\lambda: \mathbb{R} \rightarrow \mathbb{R}$ be a continuous function. The following are equivalent:

(i) There is a flow $T$ such that $\delta_{T}$ is the closure of $\lambda D \mid C_{c}^{\infty}(\mathbb{R})$,

(ii) $A(\lambda)=\varnothing$.

Proof. (i) $\Rightarrow$ (ii). For $y$ in $Z(\lambda),\left(\delta_{T} f\right)(y)=0$ for all $f$ in $C_{c}^{\infty}(\mathbb{R})$, and hence for all $f$ in $\mathscr{D}\left(\delta_{T}\right)$. It follows that $T_{t} y=y$. Thus for $x$ in $U(\lambda)$, $\left\{T_{t} x\right\} \subset U(\lambda)$, so, by Corollary 2.3, $\alpha_{x} \notin A_{r}(\lambda)$ and $\beta_{x} \notin A_{l}(\lambda)$. Now if there exists $z$ in $A_{l}(\lambda)$, then there exists $x$ in $U(\lambda)$ such that $x<z$ and $1 / \lambda$ is integrable over $(x, z)$ and therefore over $\left(x, \beta_{x}\right)$. But then $\beta_{x} \in$ $A_{l}(\lambda)$, which is a contradiction. Similarly, $A_{r}(\lambda)$ is empty.

(ii) $\Rightarrow$ (i). For $x$ in $U(\lambda)$, there is a (unique) function $q$ such that $q(x)=0$ and $q^{\prime}=1 / \lambda$ in $\left(\alpha_{x}, \beta_{x}\right) ; q$ is injective, and, by assumption, $q$ maps $\left(\alpha_{x}, \beta_{x}\right)$ onto $\mathbb{R}$. Define $T_{t} x=q^{-1}(t)$. For $y$ in $Z(\lambda)$, define $T_{t} y=y$. It is easy to verify that $T$ is a flow with speed $\lambda$.

The open set $U(\lambda)$ may be decomposed into a countable union of disjoint open intervals $\left(a_{i}, b_{i}\right)$. Let $\mathscr{D}(\lambda)$ be the algebra of all functions $f$ in $C_{c}^{1}(\mathbb{R})$ which are constant in some neighborhood of each $a_{i}$ and in some neighborhood of each $b_{i}$. Since $T$ fixes each $a_{i}$ and each $b_{i}, \mathscr{D}(\lambda)$ is invariant under the dual action of $T$-the derivative of $f \circ T_{t}$ is $\left(\lambda \circ T_{t}\right)\left(f^{\prime} \circ T_{t}\right) / \lambda$ on $U(\lambda)$. Since $\mathscr{D}(\lambda)$ is dense in $C_{0}(\mathbb{R})$, and contained in $\mathscr{D}\left(\delta_{T}\right)$, it follows that $\mathscr{D}(\lambda)$, and therefore $C_{c}^{1}(\mathbb{R})$, is a core for $\delta_{T}$. Finally, given $f$ in $C_{c}^{1}(\mathbb{R})$ with support in $[-N, N]$, there is a sequence $f_{n}$ in $C_{c}^{\infty}(\mathbb{R})$ with support in $[-N, N]$ such that $\left\|f-f_{n}\right\| \rightarrow 0,\left\|f^{\prime}-f_{n}^{\prime}\right\| \rightarrow 0$. Then $\left\|\boldsymbol{\delta}_{T} f_{n}-\boldsymbol{\delta}_{T} f\right\| \rightarrow 0$. Thus $C_{c}^{\infty}(\mathbb{R})$ is a core for $\boldsymbol{\delta}_{T}$.

If $A(\lambda) \neq \varnothing$, there may or may not be a flow with speed $\lambda$, and any such flow may or may not be unique. Suppose for example that there exists $x$ in $A_{l}^{+}(\lambda) \cap A_{r}^{-}(\lambda)$. Then any flow with speed $\lambda$ would reach $x$ from neighboring points on either side in a finite length of time, but would have no way of leaving $x$. So there is no flow with speed $\lambda$. On the other hand, if there are sufficiently many zeros of $\lambda$, a flow $T$ may be delayed at the zeros. These delays are measured by $\mu$ where

$$
\mu\left(I_{T}(x, t)\right)=|t|-\int_{I_{T}(x, t)} \frac{d y}{|\lambda(y)|}
$$

for $x$ in $U(\lambda)$, where $I_{T}(x, t)$ is the open interval between $x$ and $T_{t} x$. Since the intervals $I_{T}(x, t)$ are disjoint from the fixed point space $\mathbb{R}_{T}^{0}$, there is no restriction on $\mu$ on $\mathbb{R}_{T}^{0}$, and, for standardisation, one may as well assume that $\mu\left(\mathbb{R}_{T}^{0}\right)=0$. Thus a (positive) measure $\mu$, defined on the 
Borel subsets of $\mathbb{R}$, will be said to be a delay measure for $T$ if (1) is satisfied and $\mu\left(\mathbb{R}_{T}^{0}\right)=0$.

Conversely, it is possible to reconstruct $T$ from $\mu$ by observing that $T_{t} x=y$ if $x<y$ and

$$
\int_{x}^{y} \frac{d z}{\lambda(z)}+(\operatorname{sgn} t) \mu(x, y)=t .
$$

This sets up a bijective correspondence between flows with speed $\lambda$ and a certain class of measures, which have to be identified. A formal statement will be made in Theorem 2.5, for which the following notation and terminology is needed. As suggested above, finiteness of the delays and integrability of $1 / \lambda$ on one side of a zero of $\lambda$ has to be balanced on the other side with no change of sign of $\lambda$.

For a measure $\mu$ on $\mathbb{R}$, let $F_{l}(\mu)$ (respectively, $F_{r}(\mu)$ ) be the set of all $x$ in $(-\infty, \infty]$ (respectively, $[-\infty, \infty)$ ) for which $\mu(y, x)<\infty$ for some $y<x$ (respectively, $\mu(x, z)<\infty$ for some $z>x$ ). Then $\mu$ will be said to be a fluid measure for $\lambda$ if $\mu$ is non-atomic,

$$
A_{l}^{ \pm}(\lambda) \cap F_{l}(\mu)=A_{r}^{ \pm}(\lambda) \cap F_{r}(\mu),
$$

and $\mu$ is carried by $A_{l}(\lambda) \cap F_{l}(\mu)\left(=A_{r}(\lambda) \cap F_{r}(\mu)\right)$. Note that all these sets are Borel measurable, and that $A_{l}(\lambda) \backslash A_{r}(\lambda)$ etc. are countable and therefore null for measures $\mu$ which are non-atomic.

THEOREM 2.5. Let $\lambda: \mathbb{R} \rightarrow \mathbb{R}$ be a continuous function. For any fluid measure $\mu$ for $\lambda$, there is a unique flow $T$ on $\mathbb{R}$ with speed $\lambda$ for which $\mu$ is a delay measure. Conversely, for any flow $T$ with speed $\lambda$, there is a unique delay measure $\mu$ for $T$, and $\mu$ is a fluid measure for $\lambda$.

Proof. For simplicity, we shall write $A_{l}^{+}, F_{l}$, etc. in place of $A_{l}^{+}(\lambda)$, $F_{l}(\lambda)$ etc., and put

$$
\begin{array}{ll}
V^{+}=\{x: \lambda(x) \geq 0\}, & V^{-}=\{x: \lambda(x) \leq 0\}, \\
U^{+}=\{x: \lambda(x)>0\}, & U^{-}=\{x: \lambda(x)<0\} .
\end{array}
$$

Let $\mu$ be a fluid measure. Define an equivalence relation on $\mathbb{R}$ by saying that points $x$ and $y$ with $x<y$ are equivalent if $\mu(x, y)<\infty$ and $1 / \lambda$ is integrable over $(x, y)$. Let $C_{x}$ be the equivalence class of $x$; it is clear that $C_{x}$ is some interval in $\mathbb{R}$. If $C_{x}$ consists of the single point $x$, define $T_{t} x=x$. Otherwise, let $a$ and $b$ be the endpoints of $C_{x}$, so that $-\infty \leq a \leq x \leq b \leq \infty$. To define $T_{t} x$, the first stage is to show that $C_{x}$ is contained in $V^{+}$or in $V^{-}$. Suppose that there exist $y^{-}$in $C_{x} \cap U^{-}$and $y^{+}$ 
in $C_{x} \cap U^{+}$, and suppose for the sake of argument that $y^{-}<y$. Let $y=\sup \left(\left(y^{-}, y^{+}\right) \cap U^{+}\right)$, so that $y^{-}<y<y^{+}$. Then $\left(y, y^{+}\right)$is contained in $V^{+}$, and $y$ is equivalent to $y^{+}$, so $y \in A_{r}^{+} \cap F_{r}$. By (2), $y \in A_{l}^{+}$ which contradicts the fact that $y$ is the limit of an increasing sequence in $U^{-}$.

Now suppose for the sake of argument that $C_{x}$ is contained in $V^{+}$(the other case is similar). If $a=x$, then $x \notin A_{l}^{+} \cap F_{l}=A_{r}^{+} \cap F_{r}$, so $b=x$. Thus we need only consider the case $a<x<b$. Define

$$
\varphi\left(x^{\prime}\right)= \begin{cases}-\int_{x^{\prime}}^{x} \frac{d y}{\lambda(y)}-\mu\left(x^{\prime}, x\right) & \left(a \leq x^{\prime} \leq x\right), \\ \int_{x}^{x^{\prime}} \frac{d y}{\lambda(y)}+\mu\left(x, x^{\prime}\right) & \left(x \leq x^{\prime} \leq b\right) .\end{cases}
$$

By definition of the equivalence relation, and (2),

$$
a \notin A_{l} \cap F_{l} \supset A_{r}^{+} \cap F_{r} \text {. }
$$

Since $(a, x) \subset V^{+}$, it follows that either $\mu(a, x)=\infty$ or $\int_{a}^{x} \lambda(y)^{-1} d y=$ $\infty$, so $\varphi(a)=-\infty$. Similarly, $\varphi(b)=\infty$. In particular, neither $a$ nor $b$ is equivalent to $x$, so $C_{x}=(a, b)$.

Since $\mu$ is non-atomic, $\varphi$ is continuous, and $\varphi$ is clearly strictly increasing. Thus for each $t$ in $R$, there is a unique point $T_{t} x$ in $(a, b)$ such that $\varphi\left(T_{t} x\right)=t$, and $t \mapsto T_{t} x$ is a homeomorphism of $\mathbb{R}$ onto $(a, b)=C_{x}$. It is clear that $T_{0} x=x$ and (1) holds.

If $T$ is defined on $\mathbb{R} \times \mathbb{R}$ in this way, then for $s, t \geq 0$ and with the above notation and assumptions, using (1) with $x$ replaced by $T_{t} x$,

$$
\begin{aligned}
\varphi\left(T_{s+t} x\right) & =s+t=\int_{T_{t} x}^{T_{s} T_{t} x} \frac{d y}{\lambda(y)}+\mu\left(T_{t} x, T_{s} T_{t} x\right)+t \\
& =\varphi\left(T_{s} T_{t} x\right)-\varphi\left(T_{t} x\right)+\varphi\left(T_{t} x\right)=\varphi\left(T_{s} T_{t} x\right),
\end{aligned}
$$

so $T_{s+t} x=T_{s} T_{t} x$. Dealing similarly with other cases, it follows that $T$ satisfies the group property. Since $T_{t}$ is an order-preserving homeomorphism of each $C_{x}$, it is a homeomorphism of $\mathbb{R}$. It is clear from the construction that $t \mapsto T_{t} x$ is continuous, so $T$ is a continuous flow on $\mathbb{R}$. (For flows on $\mathbb{R}$, it is elementary to establish joint continuity from separate continuity, but flows on general spaces have the same property (see [5, Lemma 2.4]) for example).

For $x$ in $A_{l} \cap F_{l}, C_{x}$ is non-trivial, so $x$ is not fixed by $T$. Thus $A_{l} \cap F_{l}$ is disjoint from $\mathbb{R}_{T}^{0}$ (actually $\mathbb{R}_{T}^{0}=\mathbb{R} \backslash\left(A_{l} \cap F_{l}\right)$ ). Since $\mu$ is carried by $A_{l} \cap F_{l}, \mu$ is a delay measure. Since $\mu(U)=0$, it follows from (1) and the construction that Lemma 2.2(iv) is satisfied, so that $T$ has speed $\lambda$. 
Let $S$ be any flow with speed $\lambda$ for which $\mu$ is a delay measure. For $x$ in $U^{+}, S_{t} x$ increases with $t$ for small $t$ by Lemma 2.2(iv), and hence for all $t$ (since $t \mapsto S_{t} x$ is either strictly monotone or constant by the group property). Now $S_{t} x$ is determined by (1). Similarly $S_{t} x$ is uniquely determined for $x$ in $U^{-}$. Any interior point of $Z$ is fixed under $S$. Thus $S_{t} x$ is uniquely determined for all $x$ in a dense subset of $\mathbb{R}$, so by continuity $S$ is unique.

Now let $T$ be a flow with speed $\lambda$, let $x$ be a point in $\mathbb{R} \backslash \mathbb{R}_{T}^{0}$ and $C$ be the trajectory of $x$. Now $t \mapsto T_{t} x$ is injective, hence strictly monotone, and suppose for the sake of argument that it is increasing, so $C$ is contained in $V^{+}$by Lemma 2.2(i). If for some $\varepsilon>0$ and $s_{1}<s_{2}$, $\lambda\left(T_{t} x\right)<\varepsilon$ whenever $s_{1}<t<s_{2}$, then by Lemma 2.2(iv),

$$
T_{s_{2}} x-T_{s_{1}} x<\varepsilon\left(s_{2}-s_{1}\right) \text {. }
$$

For $t_{1}<t_{2},\left\{y \in\left(T_{t_{1}} x, T_{t_{2}} x\right): \lambda(y)<\varepsilon\right\}$ is a countable union of disjoint intervals of the type $\left(T_{s_{1}} x, T_{s_{2}} x\right)$, so it follows that its Lebesgue measure is less than $\varepsilon\left(t_{2}-t_{1}\right)$. Hence $Z \cap\left(T_{t_{1}} x, T_{t_{2}} x\right)$ is (Lebesgue) null.

If $\lambda\left(T_{t} x\right)>0$ whenever $s_{1}^{\prime}<t<s_{2}^{\prime}$, then by Lemma 2.2(iv),

$$
s_{2}^{\prime}-s_{1}^{\prime}=\int_{T_{s_{1}^{\prime}} x}^{T_{s_{2}^{\prime}} x} \frac{d y}{\lambda(y)} .
$$

Now $U^{+} \cap\left(T_{t_{1}} x, T_{t_{2}} x\right)$ is a countable union of disjoint intervals of the form $\left(T_{s_{1}^{\prime}} x, T_{s_{2}^{\prime}} x\right)$ and, taking the sum over these intervals and using the nullity of $Z \cap\left(T_{t_{1}} x, T_{t_{2}} x\right)$ gives

$$
t_{2}-t_{1} \geq \int_{U \cap\left(T_{t_{1}} x, T_{\left.t_{2} x\right)}\right.} \frac{d y}{\lambda(y)}=\int_{T_{t_{1}} x}^{T_{t_{2} x}} \frac{d y}{\lambda(y)} .
$$

Define a function $F_{C}$ on $C$ by

$$
F_{C}\left(T_{t} x\right)= \begin{cases}t+\int_{T_{t} x}^{x} \frac{d y}{\lambda(y)} & (t \leq 0), \\ t-\int_{x}^{T_{t} x} \frac{d y}{\lambda(y)} & (t>0) .\end{cases}
$$

Then $F_{C}$ is continuous and (4) shows that $F_{C}$ is increasing. So $F_{C}$ determines a (positive) non-atomic Lebesgue-Stieltjes measure $\mu_{C}$ on $C$, and $\mu_{C}$ may be regarded as a measure on $\mathbb{R}$. Furthermore $\mu_{C}$ is independent of the choice of $x$ in $C$, since replacing $x$ by $T_{t} x$ alters $F_{C}$ only by a constant. For $t>0$ it is immediate that

$$
\int_{x}^{T_{t} x} \frac{d y}{\lambda(y)}+\mu_{C}\left(x, T_{t} x\right)=t
$$


Also (3) shows that any compact subinterval of the open set $C \cap U^{+}$, and hence $C \cap U^{+}$itself, is $\mu_{C}$-null, so $\mu_{C}$ is carried by $C \cap Z$.

Similarly for a non-trivial trajectory $C$ contained in $V^{-}$, one may construct a non-atomic measure $\mu_{C}$, carried by $C \cap Z$, such that

$$
\int_{x}^{T_{t} x} \frac{d y}{\lambda(y)}-\mu_{C}\left(x, T_{t} x\right)=t \quad(t<0) .
$$

There are only countably many non-trivial trajectories $C$; let $\mu$ be the sum of all the corresponding measures $\mu_{C}$. It is clear that $\mu\left(\mathbb{R}_{T}^{0}\right)=0$, and (5) and (6) show that (1) also holds, so $\mu$ is a delay measure for $T$.

Suppose $x$ is a point in $Z$ with non-trivial trajectory $C$. Assuming that $C$ is contained in $V^{+},(5)$ gives

$$
\int_{T_{-1} x}^{T_{1} x} \frac{d y}{\lambda(y)}+\mu_{C}\left(T_{-1} x, T_{1} x\right)=2,
$$

so $x \in A_{l}^{+} \cap F_{l} \cap A_{r}^{+} \cap F_{r}$.

Now consider a point in $\mathbb{R}_{T}^{0} \cap A_{l}^{+}$. For all sufficiently large $x^{\prime}<x$, $\left(x^{\prime}, x\right)$ is contained in $V^{+}$and $1 / \lambda$ is integrable over $\left(x^{\prime}, x\right)$. Let $x^{\prime \prime}$ be any point of $U^{+} \cap\left(x^{\prime}, x\right)$. The trajectory $C$ of $x^{\prime \prime}$ is contained in $(-\infty, x)$, so

$$
\begin{aligned}
\mu\left(x^{\prime}, x\right) & \geq \mu_{C}\left(x^{\prime \prime}, x\right) \geq \lim _{t \rightarrow \infty} \mu_{C}\left(x^{\prime \prime}, T_{t} x^{\prime \prime}\right) \\
& =\lim _{t \rightarrow \infty}\left\{t-\int_{x^{\prime \prime}}^{T_{t} x^{\prime \prime}} \frac{d y}{\lambda(y)}\right\}=\infty,
\end{aligned}
$$

using (5) in the penultimate step. Thus $x \notin F_{l}$.

These and similar arguments show that

$$
\begin{aligned}
\left(A_{l} \cap F_{l}\right) & \cup\left(A_{r} \cap F_{r}\right) \\
& \subset Z \backslash \mathbb{R}_{T}^{0} \subset\left[\left(A_{l}^{+} \cap A_{r}^{+}\right) \cup\left(A_{l}^{-} \cap A_{r}^{-}\right)\right] \cap F_{l} \cap F_{r} .
\end{aligned}
$$

Thus $\mu$ is a fluid measure.

Finally, let $\mu^{\prime}$ be any delay measure for $T$. Then (1) shows that $\mu^{\prime}$ is uniquely determined on any open subinterval of a non-trivial trajectory, and is $\sigma$-finite on the trajectory. Hence $\mu^{\prime}$ is uniquely determined on each non-trivial trajectory. Since $\mu^{\prime}$ is carried by the union of the countable set of non-trivial trajectories, it follows that $\mu^{\prime}$ is unique. This completes the proof of Theorem 2.5.

From Theorem 2.5 , it is a routine matter of measure theory to determine those $\lambda$ for which there is a (unique) flow with speed $\lambda$. 
COROllary 2.6. There is at least one flow on $\mathbb{R}$ with speed $\lambda$ if and only if $(x, y) \cap Z(\lambda)$ is uncountable whenever $-\infty \leq x<y \leq \infty$ and either $x \in\left(A_{r}^{+}(\lambda) \backslash A_{l}^{+}(\lambda)\right) \cup\left(A_{r}^{-}(\lambda) \backslash A_{l}^{-}(\lambda)\right)$ or $y \in\left(A_{l}^{+}(\lambda) \backslash A_{r}^{-}(\lambda)\right)$ $\cup\left(A_{l}^{-}(\lambda) \backslash A_{r}^{-}(\lambda)\right)$. The flow is unique if and only if $A_{l}^{+}(\lambda)=A_{r}^{+}(\lambda)$, $A_{l}^{-}(\lambda)=A_{r}^{-}(\lambda)$ and $A(\lambda)$ is countable. If there are two distinct flows with speed $\lambda$, then there are uncountably many.

If $\overline{\lambda D \mid C_{c}^{\infty}(\mathbb{R})}$ generates a $C_{0}$-semigroup $\tau$, then the derivation law implies that $\tau_{t}$ is an endomorphism of $C_{0}(\mathbb{R})$. Since all $C_{0}$-groups of *-automorphisms arise from flows, Theorem 2.5 covers all cases when $\overline{\lambda D \mid C_{c}^{\infty}(\mathbb{R})}$ generates a $C_{0}$-group. A $C_{0}$-semigroup of endomorphisms corresponds to a half-flow $T$ on $\mathbb{R}=\mathbb{R} \cup\{ \pm \infty\}$ which fixes $\pm \infty$, that is, a continuous mapping $T: \overline{\mathbb{R}} \times[0, \infty) \rightarrow \overline{\mathbb{R}}$ such that

$$
T_{0} x=x, \quad T_{s} T_{t}=T_{s+t}, \quad T_{t} \infty=\infty, \quad T_{t}(-\infty)=-\infty .
$$

The analogue of Theorem 2.4 follows.

Proposition 2.7. Let $\lambda: \mathbb{R} \rightarrow \mathbb{R}$ be continuous. The following are equivalent:

(i) $\overline{\lambda D \mid C_{c}^{\infty}(\mathbb{R})}$ generates a $C_{0}$-semigroup on $C_{0}(\mathbb{R})$,

(ii) $A_{r}^{+}(\lambda)=A_{l}^{-}(\lambda)=\varnothing$.

The $C_{0}$-semigroup in Proposition 2.7 arises from a half-flow on $\mathbb{R}$ (as opposed to $\overline{\mathbb{R}}$ ) if and only if $-\infty \notin A_{r}^{-}(\lambda)$ and $\infty \notin A_{l}^{+}(\lambda)$, that is, $1 / \lambda$ is not integrable at $\pm \infty$.

All the results of this section have analogues for $\mathbb{T}(=\mathbb{R} / \mathbb{Z})$ and $[0,1]$, provided that $A_{l}^{+}(\lambda)$ etc. are interpreted correctly. For $\mathbb{T}$, regard $\lambda$ : $\mathbb{T} \rightarrow \mathbb{R}$ as a periodic function on $\mathbb{R}$ and let $A_{l}^{+}(\lambda)$ consist of those $x$ in $Z(\lambda)$ such that for some $y<x, \lambda \geq 0$ in $(y, x)$ and $1 / \lambda$ is integrable over $(y, x)$, etc. The statements of Theorems 2.4 and 2.5 and Proposition 2.7 are almost unchanged. For $[0,1]$, let $A_{l}^{+}(\lambda)$ consist of those $x \neq 0$ in $Z(\lambda)$ such that, for some $0<y<x, \lambda \geq 0$ in $(y, x)$ and $1 / \lambda$ is integrable over $(y, x)$; let $A_{r}^{+}(\lambda)$ consist of those $x \neq 1$ in $Z(\lambda)$ such that for some $x<z<1, \lambda \leq 0$ in $(x, z)$ and $1 / \lambda$ is integrable over $(x, z)$, etc. The statements of Theorem 2.4 become:

(i) There is a flow $T$ on $[0,1]$ such that $\delta_{T}=\overline{\lambda D}$,

(ii) $A(\lambda)=\varnothing ; \lambda(0)=\lambda(1)=0$.

Theorem 2.5 is valid, but only for functions satisfying $\lambda(0)=\lambda(1)=0$. The conditions of Proposition 2.7 are:

(i) $\overline{\lambda D}$ generates a $C_{0}$-semigroup on $C[0,1]$,

(ii) $A_{r}^{+}(\lambda)=A_{l}^{-}(\lambda)=\varnothing ; \lambda(0) \geq 0, \lambda(1) \leq 0$. 
This answers a question raised in [6]. In particular, Theorem 4 of [6] remains valid if the assumption that the derivation is well-behaved is dropped, provided that the assertion that $p(0)=p(1)=0$ is replaced by the conditions $p(0) \geq 0, p(1) \leq 0$. Some of the claims made in [6] about the example on p. 77 are incorrect, and the true position is set out below. (In comparing this paper with [6], the reader should bear in mind that there is a difference in sign conventions in defining generators.)

EXAmple $2.8[6$, p. 77]. Consider $\lambda:[0,1] \rightarrow \mathbb{R}$ defined by $\lambda(x)=$ $-2 x^{1 / 2}$. Then

$$
A_{r}^{-}(\lambda)=\{0\}, \quad A_{r}^{+}(\lambda)=A_{l}^{-}(\lambda)=A_{l}^{+}(\lambda)=\varnothing .
$$

Thus condition (ii) is satisfied, and $\overline{\lambda D}$ is the generator of the half-flow $T^{-}$, where

$$
T_{t}^{-} x=\left(\max \left(x^{1 / 2}-t, 0\right)\right)^{2} .
$$

On the other hand, $-\lambda$ does not satisfy (ii) because $-\lambda(1)<0$ and $0 \in A_{r}^{+}(-\lambda)$. The half-flow $T^{+}$defined by

$$
T_{t}^{+} x=\left(\min \left(x^{1 / 2}+t, 1\right)\right)^{2}
$$

satisfies

$$
\delta_{T^{+}} f(x)=-\lambda(x) f^{\prime}(x)
$$

for $0<x<1$, but behaves differently at both endpoints.

3. General spaces. Let $S$ be a flow on a locally compact Hausdorff space $X$, with fixed point set $X_{S}^{0}$, and let $\lambda: X \backslash X_{S}^{0} \rightarrow \mathbb{R}$ be a continuous function. The problem now is to determine conditions under which there is a flow with "speed $\lambda$ relative to $S$ ", and how such flows behave at the points of $X_{S}^{0}$. The first result interprets the relative speed in two different, but equivalent, ways.

Proposition 3.1. Let $T$ be a flow on $X$, and $\lambda: X \backslash X_{S}^{0} \rightarrow \mathbb{R}$ be a continuous function. The following are equivalent:

(i) For $\omega \in$ int $X_{S}^{0}, T_{t} \omega=\omega$; for $\omega \in X \backslash X_{S}^{0}$, there is a function $\tau_{\omega}$ : $\mathbb{R} \rightarrow \mathbb{R}$ such that $T_{t} \omega=S_{\tau_{\omega}(t)} \omega(t \in \mathbb{R})$ and $\tau_{\omega}^{\prime}(0)=\lambda(\omega)$,

(ii) If $f \in \mathscr{D}\left(\delta_{S}\right)$ and $g \in C_{0}(X)$ are such that

$$
g= \begin{cases}\lambda \delta_{S} f & \text { on } X \backslash X_{S}^{0} \\ 0 & \text { on } X_{S}^{0},\end{cases}
$$

then $f \in \mathscr{D}\left(\delta_{T}\right)$ and $\delta_{T} f=g$. 
Proof. (i) $\Rightarrow$ (ii). This is a standard argument, but the details are included for completeness. For $\omega$ in $X \backslash X_{S}^{0}$,

$$
\begin{aligned}
\lim _{t \rightarrow 0} \frac{f\left(T_{t} \omega\right)-f(\omega)}{t} & =\left.\frac{d}{d t}\left(f\left(S_{\tau_{\omega}(t)} \omega\right)\right)\right|_{t=0} \\
& =\tau_{\omega}^{\prime}(0) \delta_{S} f(\omega)=g(\omega) .
\end{aligned}
$$

Replacing $\omega$ by $T_{s} \omega$, it follows that

$$
\begin{gathered}
\left|\frac{f\left(T_{t} \omega\right)-f(\omega)}{t}-g(\omega)\right|=\left|\frac{1}{t} \int_{0}^{t}\left\{\frac{d}{d s}\left(f\left(T_{s} \omega\right)\right)-g(\omega)\right\} d s\right| \\
\leq \frac{1}{|t|} \int_{0}^{t}\left|g\left(T_{s} \omega\right)-g(\omega)\right| d s \leq \sup _{|s| \leq|t|}\left\|g \circ T_{s}-g\right\| .
\end{gathered}
$$

By continuity, this estimate remains valid for $\omega$ in $\overline{X \backslash X_{S}^{0}}$, while it is trivially valid for $\omega$ in int $X_{S}^{0}$. Thus

$$
\left\|t^{-1}\left(f \circ T_{t}-f\right)-g\right\| \leq \sup _{|s| \leq|t|}\left\|g \circ T_{s}-g\right\| \rightarrow 0 \quad \text { as } t \rightarrow 0 .
$$

Thus $f \in \mathscr{D}\left(\delta_{T}\right)$, and $\delta_{T} f=g$.

(ii) $\Rightarrow$ (i). Firstly, consider $\omega$ in $X \backslash X_{S}^{0}$. The argument used in [3] to show that $\left\{T_{t} \omega\right\} \subset\left\{S_{s} \omega\right\}$ is still valid, so there is a function $\tau_{\omega}$ such that $T_{t}=S_{\tau_{\omega}(t)} \omega$. Furthermore, $\tau_{\omega}$ is uniquely determined modulo the $S$-period of $\omega$, and one may (uniquely) arrange that $\tau_{\omega}$ is continuous and $\tau_{\omega}(0)=0$. It was shown in [4, Theorem 2.1] that there exists $f$ in $\mathscr{D}\left(\delta_{S}\right)$ such that $f\left(S_{s} \omega\right)=s$ for all small $|s|$, and $\operatorname{supp} f \subset X \backslash X_{S}^{0}$. It follows from (ii) that $f \in \mathscr{D}\left(\boldsymbol{\delta}_{T}\right)$ and

$$
\lambda(\omega)=\left(\delta_{T} f\right)(\omega)=\lim _{t \rightarrow 0} \frac{\tau_{\omega}(t)}{t}=\tau_{\omega}^{\prime}(0) .
$$

Next, for any function $h$ in $C_{0}(X)$ with supp $h$ contained in int $X_{S}^{0}$, it follows from (ii) that $h \in \mathscr{D}\left(\delta_{T}\right)$ and $\delta_{T} h=0$. The local nature of $\delta_{T}$ ensures that each point of int $X_{S}^{0}$ is fixed by $T$.

REMARK. The class $\mathscr{D}$ of functions $f$ which satisfy condition (ii) of Proposition 3.1 is a ${ }^{*}$-subalgebra of $\mathscr{D}\left(\delta_{S}\right)$, but it may not separate the points of $X_{S}^{0}$. Furthermore the flow $T$ may not fix every point of $X_{S}^{0}$ (so that $T$ may not be a "fluctuation" of $S$ in the sense of [2]). For example, let $X=\mathbb{R}^{2}, S_{t}(x, y)=(x+t y, y), T_{t}(x, y)=(x+t, y)$. Here $X_{S}^{0}=$ $\mathbb{R} \times\{0\}$ and $\lambda(x, y)=1 / y(y \neq 0)$, while $\mathscr{D}$ fails to separate any points of $X_{S}^{0}$. 
A sufficient condition that $T$ fixes each point of $X_{S}^{0}$ is condition (i) in Theorem 3.2 below (see [3] and the proof of Theorem 3.2). Sufficient conditions that $\mathscr{D}$ is a core for $\delta_{T}$ (in particular, $\mathscr{D}$ separates the points of $X$, and $T$ fixes $X_{S}^{0}$ ) were given in $[3,7,8]$.

THEOREM 3.2. Let $\lambda$ : $X \backslash X_{S}^{0}$ be a continuous function, and suppose that

(i) For any compact set $K \subset X$, there exists $\varepsilon>0$ such that $\lambda$ is bounded on $\left\{\omega \in K \backslash X_{S}^{0}: \nu(\omega)<\varepsilon\right\}$,

(ii) If $\lambda(\omega)=0$ for some $\omega$ in $X \backslash X_{S}^{0}$, then $t \mapsto \lambda\left(S_{t} \omega\right)^{-1}$ is not integrable over $(0, a)$ or over $(-a, 0)$ for any $a>0$,

(iii) For any $\omega$ in $X \backslash X_{S}^{0}, t \mapsto \lambda\left(S_{t} \omega\right)^{-1}$ is not integrable over $(0, \infty)$ or over $(-\infty, 0)$.

Then there is a unique flow $T$ on $X$ with speed $\lambda$ relative to $S$ (so that the conditions of Proposition 3.1 are valid).

Proof. For $\omega$ in $X \backslash X_{S}^{0}$, let $\lambda_{\omega}(t)=\lambda\left(S_{t} \omega\right)$. It follows from assumptions (ii) and (iii) and Theorem 2.5 that there is a unique flow $\theta_{\omega}$ on $\mathbb{R}$ with speed $\lambda_{\omega}$. This flow is characterised by the properties:

$$
\begin{aligned}
& x \text { is a fixed point of } \theta_{\omega} \Leftrightarrow \lambda\left(S_{x} \omega\right)=0, \\
& \int_{x}^{\theta_{\omega}(x, t)} \frac{d s}{\lambda\left(S_{s} \omega\right)}=t \quad \text { if } \lambda\left(S_{x} \omega\right) \neq 0 .
\end{aligned}
$$

The uniqueness of the flows, together with the relation

$$
\lambda_{S_{t} \omega}(x)=\lambda_{\omega}(x+t),
$$

ensures that the flows $\theta_{\omega}$ are coherent in the sense that

$$
\theta_{S_{t} \omega}(x, s)+t=\theta_{\omega}(x+t, s) .
$$

Let $\tau_{\omega}(t)=\theta_{\omega}(0, t)$ and

$$
T_{t} \omega= \begin{cases}S_{\tau_{\omega}(t)} \omega & \left(\omega \in X \backslash X_{S}^{0}\right), \\ \omega & \left(\omega \in X_{S}^{0}\right) .\end{cases}
$$

Then $T$ satisfies the group property $T_{s} T_{t}=T_{s+t}$.

In order to show that $T$ is a flow, it remains to show that $(\omega, t) \mapsto T_{t} \omega$ is jointly continuous. Let $\left(\omega_{\alpha}\right)$ and $\left(t_{\alpha}\right)$ be nets such that $\omega_{\alpha} \rightarrow \omega, t_{\alpha} \rightarrow t$. By passing to subnets and replacing $\lambda$ by $-\lambda$, it suffices to assume that $t_{\alpha} \geq 0$ and to consider six cases:

1. $\omega_{\alpha} \in X_{S}^{0}$;

2. $\omega_{\alpha} \in X \backslash X_{S}^{0}, \lambda\left(\omega_{\alpha}\right)=0$; 
3. $\omega_{\alpha} \in X \backslash X_{S}^{0}, \omega \in X \backslash X_{S}^{0}, \lambda\left(\omega_{\alpha}\right)>0, \quad \lambda(\omega)>0, \tau_{\omega_{\alpha}}\left(t_{\alpha}\right) \rightarrow \tau$, where $0 \leq \tau \leq \infty$;

4. $\omega_{\alpha} \in X \backslash X_{S}^{0}, \omega \in X \backslash X_{S}^{0}, \lambda\left(\omega_{\alpha}\right)>0, \lambda(\omega)=0$;

5. $\omega_{\alpha} \in X \backslash X_{S}^{0}, \omega \in X_{S}^{0}, \lambda\left(\omega_{\alpha}\right)>0, \nu\left(\omega_{\alpha}\right)>\nu$, where $\nu>0$;

6. $\omega_{\alpha} \in X \backslash X_{S}^{0}, \omega \in X_{S}^{0}, \lambda\left(\omega_{\alpha}\right)>0, \nu\left(\omega_{\alpha}\right) \rightarrow 0$.

Cases 1 and 2. Since $X_{S}^{0}$ is closed and $\lambda$ is continuous, either $\omega \in X_{S}^{0}$ or $\lambda(\omega)=0$. Thus

$$
T_{t_{\alpha}} \omega_{\alpha}=\omega_{\alpha} \rightarrow \omega=T_{t} \omega
$$

Case 3. Firstly, suppose that $\tau>\tau_{\omega}(t)$. Then, by construction of $\tau_{\omega}$, there exists $\theta$ such that $\tau_{\omega}(t)<\theta<\tau, \lambda\left(S_{s} \omega\right)>0$ for $0 \leq s \leq \theta$. Since $S$ is jointly continuous, $\lambda\left(S_{s} \omega_{\alpha}\right)^{-1} \rightarrow \lambda\left(S_{s} \omega\right)^{-1}$ as $\alpha \rightarrow \infty$ uniformly for $0 \leq s \leq \theta$, and therefore

$$
\int_{0}^{\theta} \frac{d s}{\lambda\left(S_{s} \omega_{\alpha}\right)} \rightarrow \int_{0}^{\theta} \frac{d s}{\lambda\left(S_{s} \omega\right)}
$$

But for large $\alpha, \tau_{\omega}(t)<\theta<\tau_{\omega_{\alpha}}\left(t_{\alpha}\right)$, so

$$
t_{\alpha}>\int_{0}^{\theta} \frac{d s}{\lambda\left(S_{s} \omega_{\alpha}\right)} \rightarrow \int_{0}^{\theta} \frac{d s}{\lambda\left(S_{s} \omega\right)}>t .
$$

This is a contradiction, so it follows that $\tau \leq \tau_{\omega}(t)$. For all sufficiently small $\theta^{\prime}>\tau, \lambda\left(S_{s} \omega\right)>0$ for $0 \leq s \leq \theta^{\prime}$, and the same argument as above shows that

$$
t_{\alpha} \leq \int_{0}^{\theta^{\prime}} \frac{d s}{\lambda\left(S_{\alpha} \omega_{\alpha}\right)} \rightarrow \int_{0}^{\theta^{\prime}} \frac{d s}{\lambda\left(S_{s} \omega\right)} .
$$

Hence $\theta^{\prime} \geq \tau_{\omega}(t)$. Since $\theta^{\prime}>\tau$ is arbitrarily small, it follows that $\tau \geq$ $\tau_{\omega}(t)$. Thus $\tau=\tau_{\omega}(t)$ and

$$
T_{t_{\alpha}} \omega_{\alpha}=S_{\tau_{\omega_{\alpha}}\left(t_{\alpha}\right)} \omega_{\alpha} \rightarrow S_{\tau} \omega=S_{\tau_{\omega}(t)} \omega=T_{t} \omega
$$

Case 4. By assumption (ii), for any $\eta>0, \int_{0}^{\eta}\left|\lambda\left(S_{s} \omega\right)\right|^{-1} d s=\infty$, and therefore

$$
\lim _{\varepsilon \rightarrow 0+} \int_{0}^{\eta} \frac{d s}{\left|\lambda\left(S_{s} \omega\right)\right|+\varepsilon}=\infty
$$

Since $\left(\left|\lambda\left(S_{s} \omega_{\alpha}\right)\right|+\varepsilon\right)^{-1} \rightarrow\left(\left|\lambda\left(S_{s} \omega\right)\right|+\varepsilon\right)^{-1}$ uniformly on $(0, \eta)$, it follows that

$$
\lim _{\varepsilon \rightarrow 0+} \lim _{\alpha \rightarrow \infty} \int_{0}^{\eta} \frac{d s}{\left|\lambda\left(S_{s} \omega_{\alpha}\right)\right|+\varepsilon}=\infty
$$


It follows that

$$
\lim _{\alpha \rightarrow \infty} \int_{0}^{\eta} \frac{d s}{\left|\lambda\left(S_{s} \omega_{\alpha}\right)\right|}=\infty
$$

and therefore $\tau_{\omega_{\alpha}}\left(t_{\alpha}\right)<\eta$ for large $\alpha$. Thus $\tau_{\omega_{\alpha}}\left(t_{\alpha}\right) \rightarrow 0$, so

$$
T_{t_{\alpha}} \omega_{\alpha}=S_{\tau_{\omega_{\alpha}}\left(t_{\alpha}\right)} \omega_{\alpha} \rightarrow \omega=T_{t} \omega
$$

Case 5. For each $\alpha$,

$$
\tau_{\omega_{\alpha}}\left(t_{\alpha}\right)=m_{\alpha} \nu\left(\omega_{\alpha}\right)^{-1}+\theta_{\alpha}
$$

where $m_{\alpha}$ is an integer, $0 \leq \theta_{\alpha}<\nu\left(\omega_{\alpha}\right)^{-1} \leq \nu^{-1}$. Passing to a subnet, one may assume that $\theta_{\alpha} \rightarrow \theta$. Then

$$
T_{t_{\alpha}} \omega_{\alpha}=S_{\tau_{\omega_{\alpha}}\left(t_{\alpha}\right)} \omega_{\alpha}=S_{\theta_{\alpha}} \omega_{\alpha} \rightarrow S_{\theta} \omega=\omega
$$

Case 6. Let $K$ be any compact neighbourhood of $\omega$, and let

$$
\tau_{\alpha}=\inf \left\{t>0: S_{t} \omega_{\alpha} \notin K\right\} .
$$

Supose that $\tau_{\alpha} \rightarrow \tau<\infty$. Then $S_{\tau_{\alpha}} \omega_{\alpha} \rightarrow S_{\tau} \omega=\omega$, so $\omega \in \overline{X \backslash K}$. This is a contradiction. It follows (on passing to subnets) that $\tau_{\alpha} \rightarrow \infty$.

By assumption (i), there is a constant $c$ such that $\left|\lambda\left(S_{s} \omega_{\alpha}\right)\right| \leq c$ whenever $0 \leq s \leq \tau_{\alpha}$, so that, for any $\eta>0$,

$$
\int_{0}^{\eta} \frac{d s}{\left|\lambda\left(S_{s} \omega_{\alpha}\right)\right|} \geq \frac{\eta}{c}
$$

for all sufficiently large $\alpha$. In particular, $\tau_{\omega_{\alpha}}\left(t_{\alpha}\right) \leq c t_{\alpha}$. Passing to a subnet, one may assume that $\tau_{\omega_{\alpha}}\left(t_{\alpha}\right) \rightarrow \tau<\infty$. Then

$$
T_{t_{\alpha}} \omega_{\alpha} \rightarrow S_{\tau} \omega=\omega=T_{t} \omega \text {. }
$$

It is clear that $T$ satisfies condition (i) of Proposition 3.1, and it remains only to establish uniqueness. If $\tilde{T}$ is any flow with relative speed $\lambda$, then for $\omega$ in $X \backslash X_{S}^{0}$, there is a unique continuous function $\tilde{\tau}_{\omega}: \mathbb{R} \rightarrow \mathbb{R}$ such that $\tilde{\tau}_{\omega}(0)=0$ and $T_{t} \omega=S_{\tilde{\tau}_{\omega}(t)} \omega$. Furthermore $\tau_{\omega}^{\prime}(0)=\lambda(\omega)$. The uniqueness ensures that

$$
\tilde{\tau}_{\omega}(s+t)=\tilde{\tau}_{\omega}(s)+\tilde{\tau}_{S_{\tau_{\omega}(s)} \omega}(t)
$$

and therefore there is a flow $\tilde{\theta}_{\omega}$ on $\mathbb{R}$ given by

$$
\tilde{\theta}_{\omega}(x, t)=\tilde{\tau}_{S_{x} \omega}(t)+x .
$$


Now $\tilde{\theta}_{\omega}$ has speed $\lambda_{\omega}$, and it follows from the uniqueness of flows with speed $\lambda_{\omega}$ that $\tilde{\theta}_{\omega}=\theta_{\omega}$. In particular

$$
\tilde{\tau}_{\omega}(t)=\tilde{\theta}_{\omega}(0, t)=\theta_{\omega}(0, t)=\tau_{\omega}(t),
$$

so $\tilde{T}_{t} \omega=T_{t} \omega\left(\omega \in X \backslash X_{S}^{0}\right)$.

For $\omega \in \operatorname{int} X_{S}^{0}, \tilde{T}_{t} \omega=\omega=T_{t} \omega$. Thus $\tilde{T}_{t}$ and $T_{t}$ coincide on a dense subset of $X$, and therefore $\tilde{T}=T$.

REMARK. Under the assumptions of Theorem 3.2, the algebra $\mathscr{D}$ considered in the remark following Proposition 3.1 equals $\mathscr{D}\left(\delta_{S}\right) \cap \mathscr{D}\left(\delta_{T}\right)$, but it is still unclear whether it is automatically a core for $\delta_{T}$. Let

$$
\mathscr{D}_{0}=\left\{f \in \mathscr{D}: f(S . \omega) \in \mathscr{D}\left(\lambda_{\omega}\right) \text { for all } \omega \in X \backslash X_{S}^{0},\right.
$$

$$
f \text { has compact support }\} \text {, }
$$

where $\mathscr{D}\left(\lambda_{\omega}\right)$ is as defined in the proof of Theorem 2.4. Then $\mathscr{D}_{0}$ is a

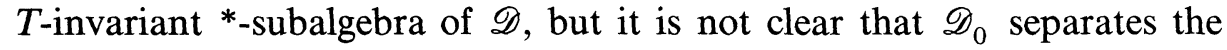
points of $X$. If so, then $\mathscr{D}$ is a core for $\delta_{T}$.

ExAMPLE 3.3. In Theorem 3.2, it is not possible to replace (ii) and (iii) by the weaker assumption

(iii)' For each $\omega$ in $X \backslash X_{S}^{0}$, there is a unique flow on $\mathbb{R}$ with speed $\lambda_{\omega}$ (where $\lambda_{\omega}(t)=\lambda\left(S_{t} \omega\right)$ ),

even if (i) is replaced by the stronger assumption that $\lambda$ is bounded. For example, let

$$
\begin{aligned}
X & =\mathbb{R} \times[0,1], \quad S_{t}(x, y)=(x+t, y) \\
\lambda(x, y) & = \begin{cases}\frac{|x|^{1 / 2}}{1+(1 / y+1)(1-|x|)^{1 / y}|x|^{1 / 2}} & (|x| \leq 1, y \neq 0), \\
|x|^{1 / 2} & (|x| \leq 1, y=0), \\
1 & (|x| \geq 1) .\end{cases}
\end{aligned}
$$

Then

$$
\int_{0}^{2} \frac{d x}{\lambda(x, 0)}=3=\int_{0}^{1} \frac{d x}{\lambda(x, y)} \quad(y \neq 0) .
$$

Since $Z\left(\lambda_{(0, y)}\right)=A_{l}^{+}\left(\lambda_{(0, y)}\right)=A_{r}^{+}\left(\lambda_{(0, y)}\right)=\{0\}$ and $A_{l}^{-}\left(\lambda_{(0, y)}\right)=$ $A_{r}^{-}\left(\lambda_{(0, y)}\right)=\varnothing$, there is a unique measure $\mu$ satisfying the conditions of Theorem 2.5 for $\lambda=\lambda_{(0, y)}$, namely $\mu=0$. The corresponding flow $\theta_{y}$ on $\mathbb{R}$ satisfies

$$
\theta_{y}(s, t)=s+\tau_{y}(t) \quad \text { where } \int_{0}^{\tau_{y}(t)} \frac{d x}{\lambda(x, y)}=t .
$$


If $T$ is any flow on $\mathbb{R}$ satisfying the conditions of Proposition 3.1, then $T$ induces flows $\tilde{\theta}_{y}$ on $\mathbb{R}$ such that

$$
T_{t}(x, y)=\left(\tilde{\theta}_{y}(x, t), y\right),
$$

and $\tilde{\theta}_{y}$ has speed $\lambda_{(0, y)}$. Hence $\tilde{\theta}_{y}=\theta_{y}$, so

$$
\begin{aligned}
& T_{3}(0, y)=\left(\tau_{y}(3), y\right)=(1, y) \quad(y \neq 0) \\
& T_{3}(0,0)=\left(\tau_{0}(3), 0\right)=(2,0) .
\end{aligned}
$$

This contradicts the continuity of $T$.

\section{REFERENCES}

[1] C. J. K. Batty, Derivations on compact spaces, Proc. London Math. Soc., (3) 42 (1981), 299-330.

[2] Delays to flows on the real line, typescript, 1980.

[3] O. Bratteli, T. Digernes, F. Goodman and D. W. Robinson, Integration in abelian $C^{*}$-dynamical systems, Publ. RIMS Kyoto Univ., 21 (1985), 1001-1030.

[4] O. Bratteli, G. A. Elliott and D. W. Robinson, The characterization of differential operators by locality: classical flows, Compos. Math., to appear.

[5] R. Derndinger and R. Nagel, Der Generator stark stetiger Verbandshalbgruppen auf $C(X)$ und dessen Spectrum, Math. Ann., 245 (1979), 159-177.

[6] R. deLaubenfels, Well-behaved derivations on $C[0,1]$, Pacific J. Math., 115 (1984), 73-80.

[7] D. W. Robinson, Smooth derivations on abelian $C^{*}$-dynamical systems, J. Austral. Math. Soc., to appear.

[8] _ Smooth cores of Lipschitz flows, Publ. RIMS Kyoto Univ., to appear.

[9] S. Sakai, Derivations in operator algebras, Studies in Appl. Math., Adv. Math. Supp. Studies, 8 (1983), 155-163, Academic Press, New York, 1983.

[10] K. S. Sibirski, Introduction to Topological Dynamics, Noordhoff, London, 1975.

[11] H. F. Trotter, Approximation and perturbation of semigroups, in: Linear operators and approximation II, P. Butzer and B. Sz. Nagy, eds., Birkhauser Verlag, Basel, 1974, pp. 3-23.

Received August 1, 1985 and in revised form February 6, 1986.

ST. JoHN's COLLEGE

OXFORD OX1 3JP

ENGLAND 



\title{
PACIFIC JOURNAL OF MATHEMATICS \\ EDITORS
}

\section{S. VARADARAJAN}

(Managing Editor)

University of California

Los Angeles, CA 90024

HERBERT CLEMENS

University of Utah

Salt Lake City, UT 84112

R. FINN

Stanford University

Stanford, CA 94305
HERMANN FLASCHKA

University of Arizona

Tucson, AZ 85721

RAMESH A. GANGOLLI

University of Washington Seattle, WA 98195

VAughan F. R. Jones

University of California

Berkeley, CA 94720

ROBION KIRBY

University of California

Berkeley, CA 94720
C. C. MOORE

University of California

Berkeley, CA 94720

H. SAMELSON

Stanford University

Stanford, CA 94305

HAROLD STARK

University of California, San Diego

La Jolla, CA 92093

\section{ASSOCIATE EDITORS}

\author{
R. ARENS \\ E. F. BECKENBACH \\ B. H. NEUMANN \\ F. WOLF \\ K. YoshIDA \\ (1906-1982)

\section{SUPPORTING INS'TITUTIONS} \\ UNIVERSITY OF ARIZONA \\ UNIVERSITY OF BRITISH COLUMBIA \\ CALIFORNIA INSTITUTE OF TECHNOLOGY \\ UNIVERSITY OF CALIFORNIA \\ MONTANA STATE UNIVERSITY \\ UNIVERSITY OF NEVADA, RENO \\ NEW MEXICO STATE UNIVERSITY \\ OREGON STATE UNIVERSITY \\ UNIVERSITY OF OREGON \\ UNIVERSITY OF SOUTHERN CALIFORNIA \\ STANFORD UNIVERSITY \\ UNIVERSITY OF HAWAII \\ UNIVERSITY OF TOKYO \\ UNIVERSITY OF UTAH \\ WASHINGTON STATE UNIVERSITY \\ UNIVERSITY OF WASHINGTON
}

The Supporting Institutions listed above contribute to the cost of publication of this Journal, but they are not owners or publishers and have no responsibility for its content or policies.

Mathematical papers intended for publication in the Pacific Journal of Mathematics should be in typed form or offset-reproduced (not dittoed), double spaced with large margins. Please do not use built up fractions in the text of the manuscript. However, you may use them in the displayed equations. Underline Greek letters in red, German in green, and script in blue. The first paragraph must be capable of being used separately as a synopsis of the entire paper. In particular it should contain no bibliographic references. Please propose a heading for the odd numbered pages of less than 35 characters. Manuscripts, in triplicate, may be sent to any one of the editors. Please classify according to the scheme of Math. Reviews, Index to Vol. 39. Supply name and address of author to whom proofs should be sent. All other communications should be addressed to the managing editor, or Elaine Barth, University of California, Los Angeles, California 90024.

There are page-charges associated with articles appearing in the Pacific Journal of Mathematics. These charges are expected to be paid by the author's University, Government Agency or Company. If the author or authors do not have access to such Institutional support these charges are waived. Single authors will receive 50 free reprints; joint authors will receive a total of 100 free reprints. Additional copies may be obtained at cost in multiples of 50 .

The Pacific Journal of Mathematics is issued monthly as of January 1966. Regular subscription rate: $\$ 190.00$ a year (5 Vols., 10 issues). Special rate: $\$ 95.00$ a year to individual members of supporting institutions.

Subscriptions, orders for numbers issued in the last three calendar years, and changes of address should be sent to Pacific Journal of Mathematics, P.O. Box 969, Carmel Valley, CA 93924, U.S.A. Old back numbers obtainable from Kraus Periodicals Co., Route 100, Millwood, NY 10546.

The Pacific Journal of Mathematics at P.O. Box 969, Carmel Valley, CA 93924 (ISSN 0030-8730) publishes 5 volumes per year. Application to mail at Second-class postage rates is pending at Carmel Valley, California, and additional mailing offices. Postmaster: send address changes to Pacific Journal of Mathematics, P.O. Box 969, Carmel Valley, CA 93924.

PUBLISHED BY PACIFIC JOURNAL OF MATHEMATICS, A NON-PROFIT CORPORATION Copyright (c) 1987 by Pacific Journal of Mathematics 


\section{Pacific Journal of Mathematics}

\section{Vol. 126, No. 2 December, 1987}

Charles James Keith Batty, Derivations on the line and flows along orbits

Paul Erdốs, Adolf J. Hildebrand, Andrew Odlyzko, Paul Pudaite and

Bruce Reznick, The asymptotic behavior of a family of sequences . . . 227

Gregory James Galloway, A note on the fundamental group of a compact

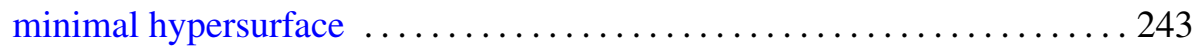

Nigel Higson, A characterization of $K K$-theory $\ldots \ldots \ldots \ldots \ldots \ldots \ldots \ldots 253$

Anthony To-Ming Lau and Wataru Takahashi, Weak convergence and nonlinear ergodic theorems for reversible semigroups of nonexpansive mappings

Pere Menal and Juame Moncasi, Lifting units in self-injective rings and an

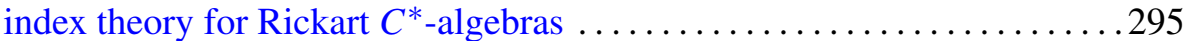

Yoshimi Saito, Schrödinger operators with a nonspherical radiation condition

Larry Smith, Realizing certain polynomial algebras as cohomology rings of

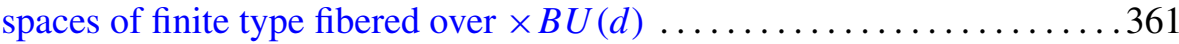

Carl E. Swenson and Calvin T. Long, Necessary and sufficient conditions for simple $A$-bases . . . . . . . . . . . . . . . . . . . . . . . . . . . . . . . 379

Kenneth Giovanni Valente, The $p$-primes of a commutative ring 385 